\title{
Feasibility and early toxicity of focal or partial brachytherapy in prostate cancer patients
}

\author{
Tae Heon Kim, MD, PhD', Jong Nyeong Kim, MD', Young Dong Yu, MD', Seung Ryeol Lee, MD, PhD!, \\ Young Kwon Hong, MD, PhD', Hyun Soo Shin, MD, PhD², Dong Soo Park, MD, PhD' \\ 'Department of Urology, CHA Bundang Medical Center, CHA University, Seongnam, Republic of Korea, ²Department of Radiation Oncology. \\ CHA Bundang Medical Center, CHA University, Seongnam, Republic of Korea
}

\begin{abstract}
Purpose: The aim of this study was to compare short-term oncologic outcomes and toxicity of focal or partial lowdose-rate brachytherapy (focal/partial LDR-BT) with whole gland low-dose-rate brachytherapy (whole LDR-BT) in localized prostate cancer patients.

Material and methods: Medical records of eligible patients who underwent focal/partial LDR-BT and whole LDR-BT between 2015 and 2017 at our institution were reviewed retrospectively. Clinical characteristics and pathologic outcomes were compared between focal/partial LDR-BT group and whole LDR-BT group. Biochemical recurrence-free survival was analyzed using Kaplan-Meier method and difference between two groups was assessed with log-rank test. Genitourinary and rectal toxicity were also evaluated between the two groups.

Results: Of the 60 patients analyzed, 30 focal/partial LDR-BT patients and 30 whole LDR-BT brachytherapy patients were included. Relative to the whole LDR-BT group, the focal/partial LDR-BT group had significantly higher initial PSA level $(p=0.002)$, smaller number of implanted seeds $(p<0.001)$, and shorter follow-up duration $(p<0.001)$. There was no significant difference between the two groups with regard to prostate volume, biopsy Gleason score, and risk group stratification. The 3-year biochemical recurrence-free survival estimates for focal/partial LDR-BT group and whole LDR-BT group were $91.8 \%$ and $89.6 \%$, respectively, which was not significantly different $(p=0.554)$. Genitourinary symptoms were significantly worse in whole LDR-BT group than in focal/partial LDR-BT group. The incidence of rectal toxicity was similar between two groups.

Conclusions: Our findings indicate that the focal/partial LDR-BT is comparable to the whole LDR-BT with respect to short-term biochemical recurrence and toxicities.

J Contemp Brachytherapy 2020; 12, 5: 420-426 DOI: https://doi.org/10.5114/jcb.2020.100374
\end{abstract}

Key words: prostate cancer, brachytherapy, focal therapy, biochemical recurrence, toxicity.

\section{Purpose}

The detection of prostate cancer has been steadily increasing worldwide, and prostate cancer is currently the most common malignancy among men [1]. Prostatespecific antigen (PSA) screening and extended prostate biopsy techniques has led to a considerable shift toward early prostate cancer. Because the prognosis for earlystage prostate cancer is favorable, the early detection of prostate cancer is associated with a significant risks of overdiagnosis and overtreatment. Majority of men treated aggressively are exposed to a significant risk of adverse effects, which might impact their quality of life more than the prostate cancer itself. The increasing awareness that men are being overtreated has led to an increased interest in focal therapy as a middle ground that aims to achieve reasonable cancer management with less toxicity, targeting only the areas of known disease within the prostate [2].
Low-dose-rate (LDR) brachytherapy is one of many effective modalities that are recommended for the curative management of men with prostate cancer. The conventional treatment of prostate cancer is based on whole gland approaches, such as radical prostatectomy and external beam radiation therapy. Similarly, the previous conventional approach in LDR brachytherapy has been based on irradiation of the entire prostate gland to a specific dose level, which provided a risk of adverse effects in terms of lower urinary tract and bowel symptoms. In response, new techniques in LDR brachytherapy were developed with advanced imaging and novel target concepts, which would be able to selectively eradicate a specific cancer focus. Zamboglou et al. [3] demonstrated that a focal brachytherapy, as a novel salvage treatment, offered a possibility for cure with a low toxicity for patients with locally recurrent prostate cancer following

Address for correspondence: Dong Soo Park, MD, PhD, Department of Urology, CHA Bundang Medical Received: 28.05 .2020 Center, CHA University, 59 Yatap-ro, Bundang-gu, 13496 Seongnam, Republic of Korea, 
radiation therapy. Especially, advancements in diagnostic tools, such as prostate mapping biopsies and multiparametric magnetic resonance imaging (MRI) significantly improved the ability to identify specific cancer lesions within the prostate [4]. Therefore, the clinician is able to treat well-defined tumoral area while sparing the remainder of gland, and focal brachytherapy can be customized to treat a specific prostate lesion at a specific dose level $[2,5,6]$. Furthermore, recently, we adopted another technique, called 'partial brachytherapy', focused on the extension of therapeutic lesion of the prostate affected by the cancer as well as the remainder of gland because of concerns about undetected tumoral area (Figure 1).

In this study, we reported our preliminary outcomes of the focal or partial brachytherapy compared to the whole gland brachytherapy, with a focus on early oncological outcome and toxicity.

\section{Material and methods}

\section{Study population}

We identified patients who underwent ${ }^{125}$ I seeds implantation LDR brachytherapy, with an implant prescription dose of 145 Gy, between January 2015 and January 2017 at our institution for the treatment of prostate cancer. Patients with incomplete data or follow-up periods shorter than 3 months were excluded. All LDR brachytherapy as focal/partial brachytherapy or whole gland brachytherapy without supplemental external beam radiation therapy were performed by a single surgeon. Details of our LDR brachytherapy technique were described previously $[7,8,9]$. The implants were preplanned using transrectal ultrasound mapping, and were subsequently performed under spinal anesthesia using transrectal ultrasound and fluoroscopy guidance. In focal/partial brachytherapy, the MRI was viewed preceding the implants, and was used to cognitively target the MRI-identified lesion. Focal brachytherapy was applied for specific cancer foci of the prostate, and the remainder of gland did not receive any treatment. On the other hand, partial brachytherapy was applied not only for cancer foci but also the remainder of prostate with additional seeds. One urologist and one radiation oncologist performed all of the implantations, using a modified peripheral iso-dose plan. The criteria for post-implant dosimetric adequacy with the volume of prostate receiving $100 \%$ and $150 \%$ of the radiation dose were $>80 \%$ and $<60 \%$, respectively. Perioperative clinicopathological data including age, initial PSA, prostate volume, clinical T stage, and biopsy Gleason score were obtained from a chart review. Gleason grading reported in this study was from a biopsy tissue. The risk group stratification was determined by D'Amico risk group classification [10], including low-risk (clinical stage T1c or T2a, preoperative PSA $10 \mathrm{ng} / \mathrm{ml}$ or less, and biopsy Gleason score 6 or less), intermediate-risk (clinical stage T2b or preoperative PSA greater than 10 and $20 \mathrm{ng} / \mathrm{ml}$ or less, or
A

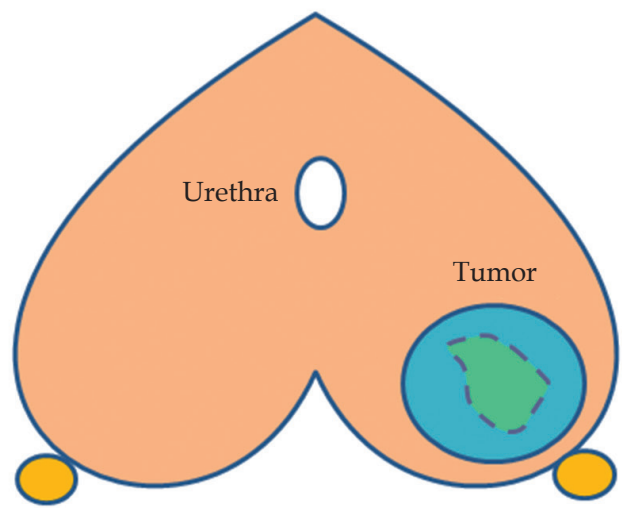

Neurovascular bundle

C

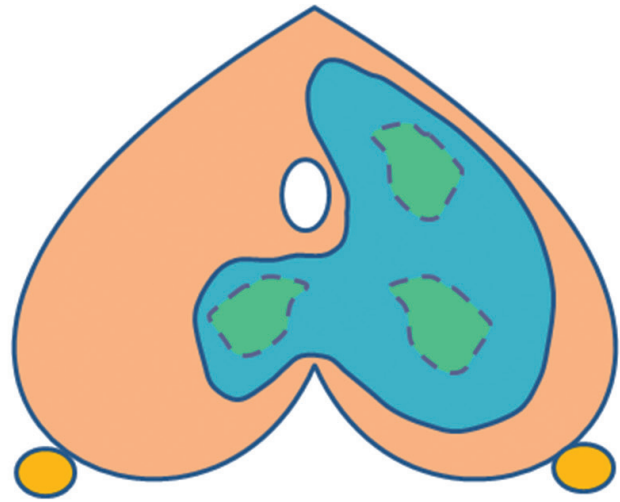

B

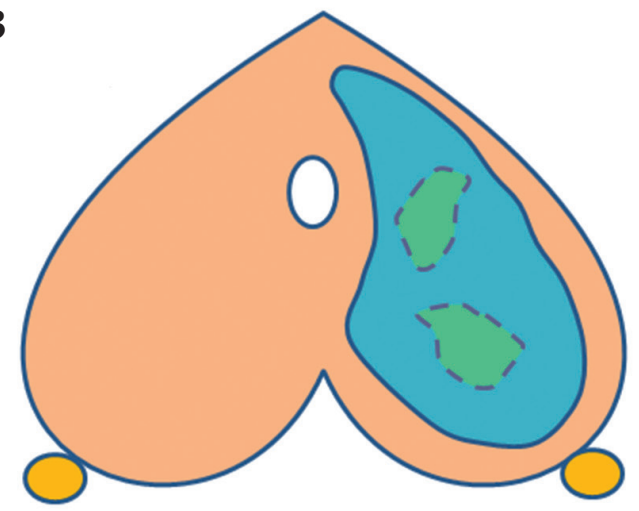

D

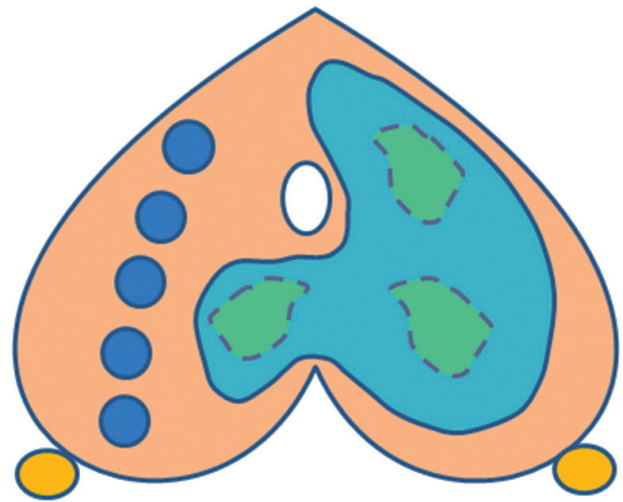

Fig. 1. Graphic illustration of the type of LDR brachytherapy according to therapeutic extent. A) Targeted brachytherapy, B) hemi-brachytherapy, C) zonal brachytherapy, D) partial brachytherapy. In this study, focal brachytherapy represent targeted brachytherapy, hemi-brachytherapy, and zonal brachytherapy 
biopsy Gleason score 7), and high-risk (clinical stage T2c or preoperative PSA $20 \mathrm{ng} / \mathrm{ml}$ or greater, or biopsy Gleason score 8-10). This retrospective study was approved by our institutional review board and the requirement for a written informed consent was waived.

\section{Study design}

Low-dose-rate brachytherapy was considered in biopsy-proven prostate cancer patients, with a Gleason score equal to or less than 8 and a total prostate volume no greater than $60 \mathrm{cc}$. The type of LDR brachytherapy as either focal/partial BT or whole gland BT was mainly determined by the patient's clinical characteristics. In gener- ally, focal brachytherapy was selected as follows: clinical stage T1c or T2a, multiparametric MRI stage T1c or T2a, PSA less than $10 \mathrm{ng} / \mathrm{ml}$, Gleason score inferior or equal to $7(3+4)$, and unilateral disease by prostate biopsy. Partial brachytherapy was selected as follows: clinical stage inferior or equal to T2c, multiparametric MRI stage T2c, PSA less than $20 \mathrm{ng} / \mathrm{ml}$, Gleason score inferior or equal to 8, and bilateral disease by prostate biopsy. Representative post-implant dosimetry images with focal brachytherapy and partial brachytherapy are shown in Figures 2 and 3. Whole gland brachytherapy was selected in remained patients, including high-risk prostate cancer patients. No patient received adjuvant androgen deprivation therapy.

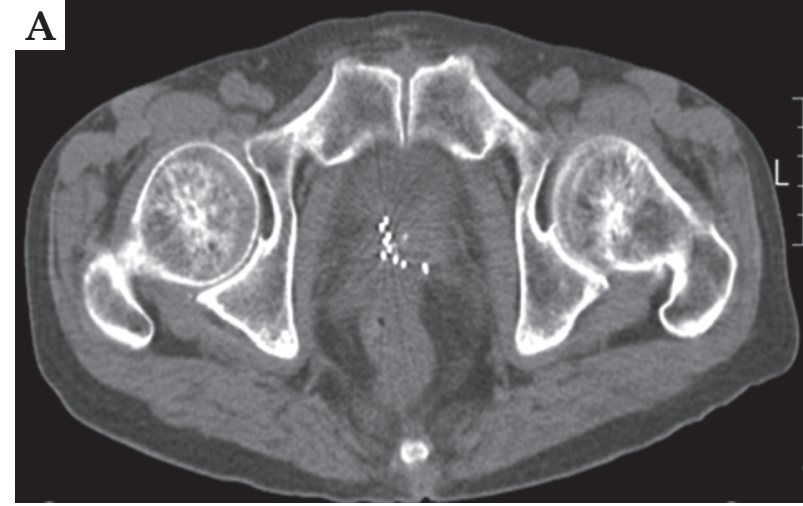

Fig. 2. Example images of focal zonal type brachytherapy of a 69-year-old patient with a prostate-specific antigen concentration of $4.8 \mathrm{ng} / \mathrm{ml}$. Digital rectal examination showed no abnormalities. A 12-core transrectal ultrasound-guided prostate biopsy revealed Gleason $3+3$ tumor in four cores in the right lobe of prostate. A) Post-implant axial computed tomography image, B) post-implant dosimetry

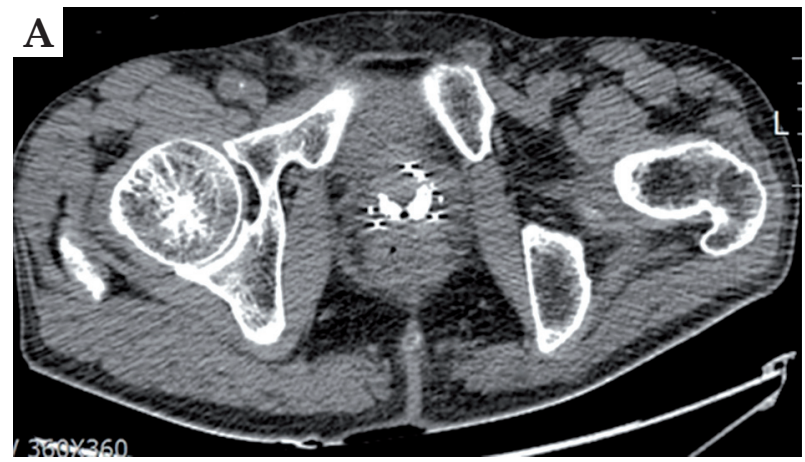

Fig. 3. Example images of partial brachytherapy of a 63-year-old patient with a prostate-specific antigen concentration of $6.0 \mathrm{ng} / \mathrm{ml}$. Digital rectal examination showed no abnormalities. A 12-core transrectal ultrasound-guided prostate biopsy revealed Gleason $3+3$ tumor in seven cores in the bilateral lobe of prostate. A) Post-implant axial computed tomography image, B) post-implant dosimetry
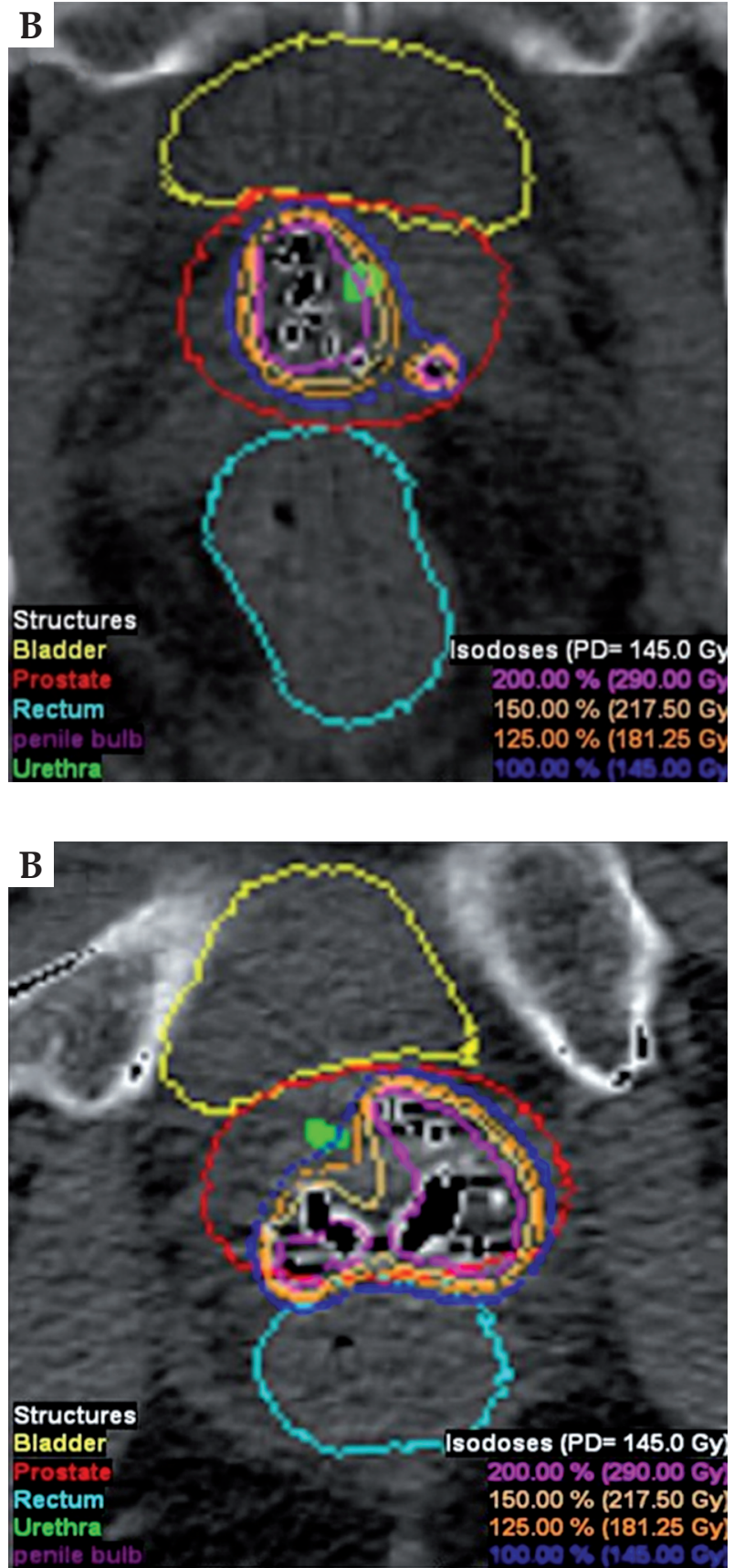
Although post-operative follow-up was not standardized due to retrospective nature of this study, the patients were generally followed-up every 3 months for the first 2 years after surgery and every 6 months thereafter. A routine checkup included digital rectal examination and serum PSA, and occurred at each visit. Multiparametric MRI, abdominal and pelvic computed tomography, and bone scans were performed if clinically indicated. Biochemical recurrence (BCR) was defined with the Radiation Therapy Oncology Group (RTOG)Phoenix definition of PSA failure as an increase in PSA by $2 \mathrm{ng} / \mathrm{ml}$ above the nadir [11]. Baseline genitourinary symptoms were recorded before the implant using international prostate symptom score (IPSS; range, 0 to 35), which consisted of questions about genitourinary symptoms. Rectal toxicity was evaluated with a modification of the RTOG morbidity scale [12]. Genitourinary and rectal toxicity were prospectively recorded and updated at each follow-up visit.

The patients were divided into two groups according to type of LDR brachytherapy: focal/partial brachytherapy or whole gland brachytherapy. Clinical and pathological variables were compared between these two groups. $\mathrm{BCR}$ and toxicity profiles were also evaluated between two groups.

\section{Statistical analysis}

Continuous variables were presented as median with interquartile range (IQR) and categorical variables as numbers with percentages. Mann-Whitney $U$ test was used to compare differences in continuous variables between the two groups. Categorical variables were compared using either Pearson's $\chi^{2}$ test or linear-by-linear association. Fisher' exact test was also applied whenever appropriate. Kaplan-Meier curves were constructed to illustrate BCR according to type of LDR brachytherapy (focal/partial brachytherapy vs. whole gland brachytherapy). The log-rank test was used to assess differences. All statistical analyses were performed using SPSS ${ }^{\circledR}$ software for Windows, version 25.0 (IBM Corp., Armonk, NY, USA). A $p$-value $<0.05$ was considered statistically significant, and all statistical tests were two-sided.

\section{Results}

\section{Baseline characteristics}

A total of 60 patients were included, comprising 30 fo$\mathrm{cal} /$ partial brachytherapy patients and 30 whole gland brachytherapy patients. Clinicopathological characteristics of patients for each group is presented in Table 1.

Table 1. Clinical characteristics of patients who underwent focal/partial or whole gland brachytherapy

\begin{tabular}{|c|c|c|c|c|}
\hline & All patients $(N=60)$ & Focal/partial BT $(n=30)$ & Whole gland BT $(n=30)$ & $P$-value \\
\hline Age (years) & $65.5(60.0-75.8)$ & $68.0(59.8-76.5)$ & $64.5(59.8-75.3)$ & 0.285 \\
\hline Initial PSA (ng/ml) & $6.7(5.1-10.5)$ & $7.6(5.5-10.3)$ & $5.9(4.7-10.9)$ & 0.002 \\
\hline Prostate volume $(\mathrm{ml})$ & $28.2(24.0-38.8)$ & $27.6(23.2-31.2)$ & $32.6(24.2-40.3)$ & 0.097 \\
\hline Clinical stage, $n(\%)$ & & & & 0.001 \\
\hline CT1 & $9(15.0)$ & $9(30.0)$ & $0(0)$ & \\
\hline cT2a & $24(40.0)$ & $13(43.3)$ & $11(36.7)$ & \\
\hline$c T 2 b$ & $9(15.0)$ & $2(6.7)$ & $7(23.3)$ & \\
\hline CT2C & $15(25.0)$ & $6(20.0)$ & $9(30.0)$ & \\
\hline сT3a & $3(5.0)$ & $0(0)$ & $3(10.0)$ & \\
\hline Biopsy Gleason (ISUP), n (\%) & & & & 0.317 \\
\hline $6(1)$ & $30(50.0)$ & $17(56.7)$ & $13(43.3)$ & \\
\hline 7 (2 or 3$)$ & $25(41.7)$ & $11(36.7)$ & $14(46.7)$ & \\
\hline $8(4)$ & $4(6.7)$ & $2(6.7)$ & $3(10.0)$ & \\
\hline D’Amico risk group, $n$ (\%) & & & & 0.098 \\
\hline Low-risk & $14(23.3)$ & $10(33.3)$ & $4(13.3)$ & \\
\hline Intermediate-risk & $41(68.3)$ & $18(60.0)$ & $23(76.7)$ & \\
\hline High-risk & $5(8.3)$ & $2(6.7)$ & $3(10.0)$ & \\
\hline Number of seeds & $45.5(40.0-59.5)$ & $40.0(37.8-40.5)$ & $59.0(53.8-65.0)$ & $<0.001$ \\
\hline Follow-up duration, months & $45.0(35.2-56.7)$ & $37.3(27.8-43.2)$ & $54.9(47.9-60.5)$ & $<0.001$ \\
\hline
\end{tabular}

All values are given as medians (interquartile ranges) or numbers (\%) of patients. BT - brachytherapy, ISUP - International Society of Urologic Pathology 
The proportions of patients who had clinical stage $\leq \mathrm{T} 2 \mathrm{a}$ in the focal/partial brachytherapy group and whole gland brachytherapy group were $73.3 \%$ and $36.7 \%$, respectively. Relative to the whole gland brachytherapy group, patients in the focal/ partial brachytherapy group had significantly higher initial PSA level $(p=0.002)$, lower clinical T stage $(p=0.001)$, smaller number of implanted seeds $(p<0.001)$, and shorter follow-up duration $(p<0.001)$. There was no significant difference between the two groups with regard to prostate volume, biopsy Gleason score, and risk group stratification.

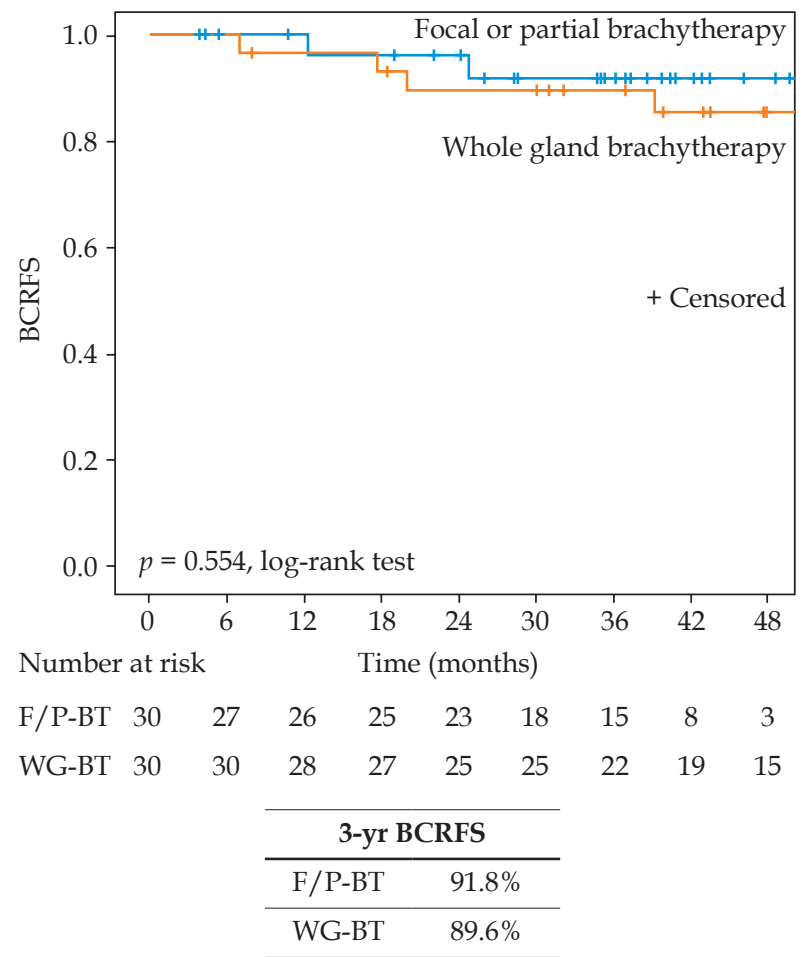

Fig. 4. Estimated biochemical recurrence-free survival stratified by focal or partial brachytherapy vs. whole gland brachytherapy

BCRFS - biochemical recurrence-free survival, F/P-BT - focal or partial brachytherapy, WG-BT-whole gland brachytherapy

\section{Oncological and clinical outcomes}

Overall, the median follow-up duration was 45.0 (IQR, 35.2-56.7) months. The proportion of patients who had reached more than 3-year follow-up was $60.0 \%(18 / 30)$ and $86.7 \%(26 / 30)$ for the focal/partial and whole gland brachytherapy groups, respectively. During follow-up, there were $6(10.0 \%)$ BCRs, including $2(6.7 \%)$ in the fo$\mathrm{cal} /$ partial brachytherapy group and $4(13.3 \%)$ in the whole gland brachytherapy group. The 3-year BCR-free survival estimates for the focal/partial brachytherapy group and whole gland brachytherapy group were $91.8 \%$ and $89.6 \%$, respectively, which was not significantly different $(p=0.554)$ (Figure 4).

The main toxicity parameters are shown in Table 2. The median initial IPSS in the focal/partial brachytherapy group and whole gland brachytherapy group was similar as 8.0 (IQR, 5.0-13.3) and 9.5 (IQR, 5.0-13.0), respectively $(p=0.499)$. Six months after the implantation, the median IPSS was found to increase to 14.0 (IQR, 9.3-16.3) and 15.0 (IQR, 9.5-19.0) in the focal/partial brachytherapy group and whole gland brachytherapy group, respectively, which was indicative of symptom worsening in both groups. The changes from baseline to 6 months in the IPSS were significantly greater in the whole gland brachytherapy group compared with the fo$\mathrm{cal} /$ partial brachytherapy group $(p=0.018)$. As for rectal toxicity, the total of 15 patients $(25.0 \%)$ experienced grade 1 or grade 2 rectal toxicity, including 5 in the focal/ partial brachytherapy group and 10 in the whole gland brachytherapy group according to the modified RTOG toxicity scale. However, there was no rectal toxicity of $\geq$ grade 3 in both the groups, and the incidence of rectal toxicity was similar between the two groups $(p=0.136)$.

\section{Discussion}

In this study, we evaluated the BCR-free survival and toxicities of the focal or partial brachytherapy for the treatment of localized prostate cancer. Our study demonstrated that short-term BCR-free survival obtained after the focal or partial brachytherapy was comparable to that of the whole gland brachytherapy for the management of patients with localized prostate cancer. Although there

Table 2. Urinary toxicity (IPSS) and rectal toxicity (RTOG grade) for focal/partial or whole gland brachytherapy

\begin{tabular}{lccc} 
& Focal/partial BT $(n=30)$ & Whole gland BT $(n=30)$ & $P$-value \\
\hline Initial IPSS & $8.0(5.0-13.3)$ & $9.5(5.0-13.0)$ & 0.499 \\
\hline IPSS at 6-month & $14.0(9.3-16.0)$ & $15.0(9.5-19.0)$ & 0.150 \\
\hline Change & $3.0(1.0-6.0)$ & $5.0(2.8-8.0)$ & 0.018 \\
\hline$P$-value & $<0.001$ & $<0.001$ & 0.136 \\
\hline Rectal toxicity, $n(\%)$ & $5(16.7)$ & $10(33.3)$ & 8 \\
\hline RTOG grade 1 & 3 & 2 &
\end{tabular}

All values are given as medians (interquartile ranges) or numbers (\%) of patients. BT - brachytherapy, IPSS - International Prostate Symptom Score, RTOG - Radiation Therapy Oncology Group, *vs. initial IPSS within treatment group 
was no significant difference, the 3-year BCR-free survival for focal/partial brachytherapy was slightly higher than that for the whole gland brachytherapy (91.8\% vs. $89.6 \%)$. Regarding toxicities, the incidence of rectal toxicity was similar between focal/partial brachytherapy group and the whole gland brachytherapy group, but genitourinary symptoms assessed using IPSS were significantly worse in whole gland brachytherapy group than in the focal/ partial brachytherapy group.

The conventional treatment of prostate cancer is based on whole gland approaches. With better quality of imaging, there is a growing interest in investigating risk adaptive strategies and several treatment approaches allowing a more favorable morbidity profile. As a result, many clinicians and patients with localized prostate cancer, face a difficult choice, i.e., active surveillance and curative treatment. The active surveillance might be related to psychosocial and financial burdens for participating patients [13]. On the other hand, the curative treatment, such as radical prostatectomy and external beam radiation therapy, can be associated with significant rates of genitourinary and rectal complications due to their side effects on adjacent structures. Targeted focal therapy is a potential bridge between the active surveillance and curative treatment modalities for patients with localized prostate cancer. Targeted focal therapy is defined as complete ablation of all clinically significant cancer foci within the prostate, using a minimally invasive technique with preservation of the sphincter, normal gland tissue, and neurovascular bundles [14]. The fundamental aspect of focal therapy is based on the targeted destruction of tumoral lesion with the preservation of surrounding healthy parenchyma [2]. There are numerous options for focal therapy, which may minimize complications related to whole gland approach, provided that the comparable oncological efficacy is maintained [15].

LDR brachytherapy treats the prostate cancer tissue from the inside, and the radiation does not travel through normal tissue to reach the target lesion as in external beam radiation therapy. It can offer the favorable dose distribution to surrounding nearby healthy tissues. LDR brachytherapy has been a reasonable approach, given its convenience, cost-effectiveness, and favorable side-effect profile $[16,17]$. Although, conventional LDR brachytherapy irradiate the whole prostate gland, focal LDR brachytherapy is rapidly attaining a worldwide interest $[18,19]$. Focal LDR brachytherapy concepts generally include targeted ablation, hemi-ablation, and zonal ablation (Figure 1). A pilot study of 21 patients by Cosset et al. [5] reported that focal brachytherapy, which ablated only index lesions was easy and feasible, with only little acute toxicity. Brun et al. [20] also demonstrated feasibility of ultra-focal brachytherapy with multiparametric MRI as able to accurately deliver high-dose on ultra-focal volume. However, these studies precluded any definitive data about oncological outcomes such as BCR-free survival. In contrary, our pilot study with focal/partial LDR brachytherapy showed comparable BCR-free survival.

Although outcomes of focal LDR brachytherapy are excellent in patients with prostate cancer, especially for low-risk prostate cancer, much of the controversy revolves on the appropriate selection of patients for a whole gland approach versus targeted focal approach. Since prostate cancer is usually multifocal, the whole gland treatment has been a standard practice. On the other hand, focal therapy usually address the index lesion as the targeted tumor and focus the treatment on malignant areas within the prostate only, preserving normal prostate and surrounding structures. However, limitations in imaging accuracy preclude the correct evaluation of the number and extension of prostate cancer target lesions [15]. In focal brachytherapy, there are certain technical challenges in application of this modality due to insufficient data of clear guidelines on isotope selection, target delineation, treatment planning, dose prescription, and treatment delivery [21]. Furthermore, there are significant concerns regarding the possibility of undertreatment of the rest of the gland due to non-visible lesions and unavoidable sampling errors in prostate biopsy related to the multifocal nature of prostate cancer. In response, we introduced partial LDR brachytherapy concepts, in which the treatment can be performed partially as more effective than zonal ablation in carefully selected patients (Figure 1D). In partial LDR brachytherapy, we included several seeds in normal prostate area, since it may accommodate the other foci of tumor or adjuvant lesions. It could help to reduce side effects with reasonable oncological outcomes, such as our preliminary rThis study has some other limitations. First, due to the retrospective and nonrandomized study design, unidentified and confounding variables and selection bias could be present. Second, it included a relatively small number of patients at a single institution, which is likely to limit the statistical aspect of the study. Future work will focus on expanding the cohort. Third, the median follow-up time was short for a detailed analysis of oncological outcomes.

\section{Conclusions}

Our results showed that the focal or partial brachytherapy was comparable to the whole gland LDR brachytherapy, with respect to short-term BCR and toxicities. Our findings suggest that focal or partial brachytherapy is a feasible technique for the treatment of patients with localized prostate cancer. Clinical outcomes of larger studies and long-term follow-up are required to determine that focal or partial brachytherapy can control localized prostate cancer with reduced early- and long-term toxicity.

\section{Disclosure}

The authors report no conflict of interest.

\section{References}

1. Siegel RL, Miller KD, Jemal A. Cancer statistics, 2020. CA Cancer J Clin 2020; 70: 7-30.

2. Perera M, Krishnananthan N, Lindner U et al. An update on focal therapy for prostate cancer. Nat Rev Urol 2016; 13: 641-653.

3. Zamboglou C, Rischke HC, Meyer PT et al. Single fraction multimodal image guided focal salvage high-dose- 
rate brachytherapy for recurrent prostate cancer. J Contemp Brachytherapy 2016; 8: 241-248.

4. Bauman G, Haider M, Van der Heide UA et al. Boosting imaging defined dominant prostatic tumors: a systematic review. Radiother Oncol 2013; 107: 274-281.

5. Cosset JM, Cathelineau X, Wakil G et al. Focal brachytherapy for selected low-risk prostate cancers: a pilot study. Brachytherapy 2013; 12: 331-337.

6. Laing R, Franklin A, Uribe J et al. Hemi-gland focal low dose rate prostate brachytherapy: An analysis of dosimetric outcomes. Radiother Oncol 2016; 121: 310-315.

7. Park DS, Gong IH, Choi DK et al. Radical prostatectomy versus high dose permanent prostate brachytherapy using iodine-125 seeds for patients with high risk prostate cancer: a matched cohort analysis. World J Urol 2013; 31: 1511-1517.

8. Park DS, Gong IH, Choi DK et al. Outcomes of Gleason Score $\leq 8$ among high risk prostate cancer treated with 125I low dose rate brachytherapy based multimodal therapy. Yonsei Med J 2013; 54: 1207-1213.

9. Yu YD, Kang MH, Choi CI et al. Clinical efficacy of combination therapy with an alpha blocker and low-dose sildenafil on post-therapy lower urinary tract symptoms after lowdose-rate brachytherapy for prostate cancer. World J Urol 2016; 34: 1269-1274.

10. D'Amico AV, Whittington R, Malkowicz SB et al. Biochemical outcome after radical prostatectomy, external beam radiation therapy, or interstitial radiation therapy for clinically localized prostate cancer. JAMA 1998; 280: 969-974.

11. Roach M 3rd, Hanks G, Thames H Jr. et al. Defining biochemical failure following radiotherapy with or without hormonal therapy in men with clinically localized prostate cancer: recommendations of the RTOG-ASTRO Phoenix Consensus Conference. Int J Radiat Oncol Biol Phys 2006; 65: 965-974.

12. Lawton CA, Won M, Pilepich MV et al. Long-term treatment sequelae following external beam irradiation for adenocarcinoma of the prostate: analysis of RTOG studies 7506 and 7706. Int J Radiat Oncol Biol Phys 1991; 21: 935-939.

13. Weerakoon M, Papa N, Lawrentschuk $\mathrm{N}$ et al. The current use of active surveillance in an Australian cohort of men: a pattern of care analysis from the Victorian Prostate Cancer Registry. BJU Int 2015; 115 Suppl 5: 50-56.

14. Crawford ED, Barqawi A. Targeted focal therapy: a minimally invasive ablation technique for early prostate cancer. Oncology (Williston Park) 2007; 21: 27-32; discussion 33-34, 39.

15. van der Poel HG, van den Bergh RCN, Briers E et al. Focal therapy in primary localised prostate cancer: the European Association of Urology Position in 2018. Eur Urol 2018; 74: 84-91.

16. Tanderup K, Menard C, Polgar C et al. Advancements in brachytherapy. Adv Drug Deliv Rev 2017; 109: 15-25.

17. Stish BJ, Davis BJ, Mynderse LA et al. Low dose rate prostate brachytherapy. Transl Androl Urol 2018; 7: 341-356.

18. Mahdavi SS, Spadinger IT, Salcudean SE et al. Focal application of low-dose-rate brachytherapy for prostate cancer: a pilot study. J Contemp Brachytherapy 2017; 9: 197-208.

19. Crook JM, Zhang P, Pisansky TM et al. A prospective phase 2 trial of transperineal ultrasound-guided brachytherapy for locally recurrent prostate cancer after external beam radiation therapy (NRG Oncology/RTOG-0526). Int J Radiat Oncol Biol Phys 2019; 103: 335-343.

20. Brun T, Bachaud JM, Graff-Cailleaud P et al. New approach of ultra-focal brachytherapy for low- and intermediate-risk prostate cancer with custom-linked I-125 seeds: A feasibility study of optimal dose coverage. Brachytherapy 2018; 17: 544-555.

21. Haworth A, Williams S. Focal therapy for prostate cancer: the technical challenges. J Contemp Brachytherapy 2017; 9: 383-389. 